\title{
SOME INTEGRAL FORMULAS FOR CLOSED HYPERSURFACES IN EUCLIDEAN SPACE
}

\author{
JAMAL K. SHAHIN
}

Introduction. Let $V^{n}$ be the orientable hypersurface twice differentiably imbedded in a Euclidean space $E^{n+1}$ of $n+1 \geqq 3$ dimensions, and let $k_{1}, \cdots, k_{n}$ be the $n$ principal curvatures at a point $P$ of $V^{n}$. The $r$ th mean curvature $H_{r}$ of $V^{n}$ at the point $P$ is defined to be the $r$ th elementary symmetric function of $k_{1}, \cdots, k_{n}$ divided by the number of terms, that is,

$$
\left(\begin{array}{l}
n \\
r
\end{array}\right) H_{r}=\sum_{i_{1}<\cdots<i_{r}} k_{i_{1}} \cdots k_{i_{r}}
$$

and $H_{0}=1$. Throughout this paper all Latin indices take the values $1, \cdots, n$. Greek indices the values $1, \cdots, n+1$, and we shall also follow the convention that repeated indices imply summation. Let $p$ denote the oriented distance from a fixed point $O$ in $E^{n+1}$ to the tangent hyperplane $\pi(P)$ of $V^{n}$ at the point $P$, and let $d \Omega$ be the area element of $V^{n}$ at $P$. Let $e_{1}, \cdots, e_{n}$ be an oriented orthonormal frame in the tangent space of the hypersurface $V^{n}$ at the point $P$, and denote by $z_{i}$ the scalar product of $e_{i}$ and the position vector of the point $P$ with respect to the fixed point $O$ in the space $E^{n+1}$. The purpose of this paper is to establish the following

THEOREM. Let $V^{n}$ be a closed orientable hypersurface twice differentiably imbedded in a Euclidean space $E^{n+1}$ of $n+1 \geqq 3$ dimensions. Then

$$
\begin{aligned}
n \int_{v^{n}} p^{m-1}\left(1+p H_{1}\right) d \Omega-(m-1) \int_{v^{n}} p^{m-2} \sum_{i, j} a_{i j} z_{i} z_{j} d \Omega & =0, \\
n \int_{v^{n}} p^{m-1}\left(H_{n-1}+p H_{n}\right) d \Omega-(m-1) \int_{v^{n}} p^{m-2} H_{n} \sum_{i} z_{i}^{2} d \Omega & =0,
\end{aligned}
$$

where $m$ is any real number, and $a_{i j}$ the coefficients of the second fundamental form of the hypersurface $V^{n}$ at a general point.

These formulas (2), (3) were obtained by Chern [2] for $n=2$, by Hsiung [3], [4] for $m=1$ in a Euclidean space $E^{n+1}$ as well as a Riemannian space $R^{n+1}$, and jointly by Hsiung and the author [5] in an affine space $A^{n+1}$.

Received by the editors January 30, 1967. 
Let $p^{\prime}$ be the foot of the perpendicular from the fixed point $O$ to the tangent hyperplane $\pi(P), d_{P}$ the distance $P P^{\prime}$ and $\rho_{P}$ the normal curvature of the hypersurface $V^{n}$ in the direction $P P^{\prime}$. Then $O P+P P^{\prime}$ $=O P^{\prime}$. By taking scalar products of both sides of this vector equation with $e_{i}$ we obtain $z_{i}+e_{i} \cdot P P^{\prime}=0$, which enables us to write $-P P^{\prime}$ $=\sum_{i} z_{i} e_{i}$. Hence we arrive at the following geometric meanings of the terms $\sum_{i} z_{1}^{2}, \sum_{i, j} a_{i j} z_{i} z_{j}$ in the formulas (3), (2):

$$
d_{p}^{2}=\sum_{i} z_{i}^{2}, \quad d_{p}^{2} \rho_{P}=\sum_{i, j} a_{i j} z_{i} z_{j}
$$

1. Preliminaries. In a Euclidean space $E^{n+1}$ of dimension $n+1 \geqq 3$ let us consider a fixed right-handed rectangular frame $Y e_{1} \cdots e_{n+1}$, where $Y$ is a point in the space $E^{n+1}$, and $e_{1}, \cdots, e_{n+1}$ are an ordered set of mutually orthogonal unit vectors such that its determinant is

$$
\left|e_{1}, \cdots, e_{n+1}\right|=1
$$

so that

$$
e_{i} e_{j}=\delta_{i j},
$$

where $\delta_{i j}$ are the Kronecker deltas. We also use $Y$ to denote the position vector of the point $P$ with respect to a fixed point $O$ in the space $E^{n+1}$. Then we can have

$$
\begin{aligned}
d Y & =\omega^{\alpha} e_{\alpha}, \\
d e_{\alpha} & =\omega_{\alpha}^{\beta} e_{\beta},
\end{aligned}
$$

where $d$ denotes the exterior differentiation, and $\omega^{\alpha}, \omega_{\alpha}^{\beta}$ are Pfaffian forms. Since $d^{2} Y=d(d Y)=d\left(d e_{\alpha}\right)=0$, exterior differentiation of equations (5), (6), (7) gives

$$
\stackrel{\beta}{\omega_{\alpha}^{\beta}}+\underset{\omega_{\beta}}{\alpha}=0,
$$

and the equations of structure of the group of proper motions in the space $E^{n+1}$ :

$$
\begin{aligned}
& d \omega^{\alpha}=\omega^{\beta} \wedge \omega_{\beta}^{\alpha}, \\
& d \omega_{\alpha}^{\beta}=\omega_{\alpha}^{\gamma} \wedge \omega_{\gamma}^{\beta},
\end{aligned}
$$

where $\wedge$ denotes the exterior product.

Let $V^{n}$ be a hypersurface twice differentiably imbedded in the space $E^{n+1}$. Consider the subfamily of frames $Y e_{1} \cdots e_{n+1}$ satisfying the conditions (i) $Y \in V^{n}$, (ii) $e_{1}, \cdots, e_{n}$ are vectors tangent to $V^{n}$ at $P$. Then we have

$$
\omega^{n+1}=0,
$$


and equations (9), (8) give

$$
\omega^{i} \wedge \omega_{i}^{n+1}=0 .
$$

By a lemma of E. Cartan [1, p. 11] on exterior algebra, equation (12) implies that for each value of $i$

$$
\omega_{i}^{n+1}=a_{i j} \omega^{j},
$$

with $a_{i j}=a_{j i}$. From equations (6), (7), (8), (13) it follows that at the point $P$ of the hypersurface $V^{n}$, the first and second fundamental forms are respectively given by

$$
\begin{gathered}
d Y \cdot d Y=\sum_{i=1}^{n}\left(\omega^{i}\right)^{2}, \\
\Phi=-d Y \cdot d e_{n+1}=a_{i j} \omega^{i} \omega^{j},
\end{gathered}
$$

and the element of area is given by

$$
d \Omega=\omega^{1} \wedge \cdots \wedge \omega^{n} .
$$

Thus the $n$ principal curvatures $k_{1}, \cdots, k_{n}$ of the hypersurface $V^{n}$ at the point $P$ are roots of the determinant equation

$$
\left|a_{i j}-k \delta_{i j}\right|=0 \text {. }
$$

In other words, $k_{1}, \cdots, k_{n}$ are the characteristic roots of the matrix $\left\|a_{i j}\right\|$.

A principal minor of the matrix $\left\|a_{i j}\right\|$ is a minor whose diagonal is part of the main diagonal of the matrix $\left\|a_{i j}\right\|$. From a theorem in linear algebra it is known that the $r$ th elementary symmetric function of the characteristic roots of the matrix $\left\|a_{i j}\right\|$ is equal to the sum of all $r$-rowed principal minors of the matrix $\left\|a_{i j}\right\|$. Hence $C_{n, r} H_{r}$ defined by equation (1) is equal to the sum of all $r$-rowed principal minors of the matrix $\left\|a_{i j}\right\|$. In particular we have

$$
\begin{aligned}
n H_{1} & =\sum_{i=1}^{n} a_{i i}, \\
H_{n} & =\left|a_{i j}\right|, \\
n H_{n-1} & =\sum_{i=1}^{n} A^{i i},
\end{aligned}
$$

where $A^{i i}$ is the cofactor of $a_{i i}$ in the determinant $\left|a_{i j}\right|$.

2. Proof of the Theorem. Let us now consider the scalar products

$$
p=Y e_{n+1}, \quad z_{\alpha}=Y e_{\alpha} .
$$


Geometrically, $z_{\alpha}$ is the oriented distance from the origin $O$ to the hyperplane $P e_{1} \cdots \hat{e}_{\alpha} \cdots e_{n+1}$, where the circumflex over $e_{\alpha}$ indicates the vector $e_{\alpha}$ is to be deleted. In particular $z_{n+1}$ will also be written as $p$. From equations (21), (7), (8), (13) follows immediately

$$
d p=-\sum_{i} a_{i j} \omega^{j} z_{i}
$$

It is convenient to write equations (21) as

$$
\begin{aligned}
p & =(-1)^{n}\left|Y, e_{1}, \cdots, e_{n}\right|, \\
z_{\alpha} & =(-1)^{\alpha-1}\left|Y, e_{1}, \cdots, \hat{e}_{\alpha}, \cdots, e_{n+1}\right| .
\end{aligned}
$$

By means of the relation $d^{2} Y=d^{2} e_{n+1}=0$ and the ordinary rule for differentiation of determinants, we have the differential forms

$$
\begin{aligned}
& d\left(p^{m-1}\left|Y, e_{n+1}, d Y, \cdots, d Y\right|\right) \quad \text { (where } d Y \text { occurs }(n-1) \text { times) } \\
& =(m-1) p^{m-2} d p \wedge\left|Y, e_{n+1}, d Y, \cdots, d Y\right| \\
& +p^{m-1}\left|Y, d e_{n+1}, d Y, \cdots, d Y\right| \\
& \text { (where } d Y \text { occurs }(n-1) \text { times), } \\
& d\left(p^{m-1}\left|Y, e_{n+1}, d e_{n+1}, \cdots, d e_{n+1}\right|\right) \\
& =(m-1) p^{m-2} d p \wedge\left|Y, e_{n+1}, d e_{n+1}, \cdots, d e_{n+1}\right| \\
& +p^{m-1}\left|d Y, e_{n+1}, d e_{n+1}, \cdots, d e_{n+1}\right|
\end{aligned}
$$

Using the elementary identity $C_{n, r}=n ! /[(n-r) ! r !]$ and equations (22), (6), (7), (8), (19), (20), (16), (23), (24), from equations (25), (26) we can easily obtain

$$
\frac{(-1)^{n-1}}{(n-1) !} d\left(p^{m-1}\left|Y, e_{n+1}, d Y, \cdots, d Y\right|\right)
$$

(where $d Y$ occurs $(n-1)$ times)

$$
=n p^{m-1}\left(1+p H_{1}\right) d \Omega-(m-1) p^{m-2} \sum_{i, j} a_{i j} z_{i} z_{j} d \Omega,
$$




$$
\begin{aligned}
& \frac{1}{(n-1) !} d\left(p^{m-1}\left|Y, e_{n+1}, d e_{n+1}, \cdots, d e_{n+1}\right|\right) \\
& \quad \text { (where } d e_{n+1} \text { occurs }(n-1) \text { times) } \\
& =n p^{m-1}\left(H_{n-1}+p H_{n}\right) d \Omega-(m-1) p^{m-2} H_{n} \sum_{i} z_{i}^{2} d \Omega .
\end{aligned}
$$

Application of Stokes' theorem to equations (27), (28) gives immediately the required formulas (2), (3).

\section{REFERENCES}

1. E. Cartan, Les systèmes différentiels extérieurs et leurs applications géométriques, Actualités Sci. Ind., No. 994, Hermann, Paris, 1945.

2. S. S. Chern, Some formulas in the theory of surfaces, Bol. Soc. Mat. Mexicana 10 (1953), 30-40.

3. C. C. Hsiung, Some integral formulas for closed hypersurfaces, Math. Scand. 2 (1954), 286-294.

4. - Some integral formulas for closed hypersurfaces in Riemannian space, Pacific J. Math. 6 (1956), 291-299.

5. C. C. Hsiung and J. K. Shahin, Affine differential geometry of closed hypersurfaces, Proc. London Math. Soc. (3) 17 (1967), 715-735.

\section{LEHIGH UNIVERSITY AND}

Salem State College 